\title{
Impacto del uso de anillos y uñas esmaltadas en la calidad de la higiene de manos en el personal de salud
}

\author{
Impact of the use of rings and nail polish on hand hygiene quality in healthcare workers
}

\author{
Felipe Olivares A. ${ }^{1}$, Teresa Vergara², Elena Véliz² y Jeannette Dabanch²
}

'Universidad de Valparaíso. Valparaíso, Chile.

${ }^{2}$ Unidad de Infecciones Asociadas a la Atención en Salud, Hospital Militar de Santiago. Santiago, Chile.

Conflicto de interés: ninguno.

Financiamiento: ninguno.

Recibido: 6 de diciembre de 2018 / Aceptado: 2 de enero de 2020

\section{Resumen}

Introducción: Las infecciones asociadas a la atención de salud son un problema frecuente en el ambiente hospitalario. La higiene de manos es la medida más efectiva para su prevención. El uso de ciertos accesorios en las manos podría disminuir su efectividad y favorecer la transmisión horizontal de agentes infecciosos. Objetivo: Revisar los estudios publicados que evalúan el impacto del uso de anillos y uñas esmaltadas en la calidad de la higiene de manos en trabajadores de la salud. Métodos: Búsqueda no sistemática en base de datos PUBMED/ MEDLINE (1978-2018) de estudios en los cuales se mide la calidad de la higiene de manos o lavado quirúrgico, mediante cultivos cuantitativos o tinciones fluorescentes. Resultados: Uso de anillos: Trece de 51 artículos cumplían los criterios de inclusión. Siete fueron realizados en unidades clínicas, y en todos ellos éste se asoció a menor calidad de la higiene de manos (la mayoría de baja calidad). Contrariamente, en tres de cuatro estudios primarios realizados en pabellón (de baja calidad), su uso no impactó en la calidad del lavado quirúrgico. Igualmente, dos revisiones sistemáticas obtuvieron similares conclusiones. Uñas esmaltadas: siete de 54 artículos fueron incluidos. En cuatro hubo resultados discordantes (la mayoría de baja calidad). En un estudio controlado se observó reducción en la calidad del lavado quirúrgico sólo cuando el esmalte estaba dañado. El esmalte gel se asoció a menor calidad de la higiene de manos en dos estudios experimentales. Conclusiones: No existe evidencia de calidad suficiente para asociar el uso de estos accesorios con reducción en la calidad de la higiene de manos. Tampoco queda demostrada su inocuidad. En base a la evidencia disponible (la mayoría de baja calidad), se observó un impacto negativo del uso de anillos en unidades clínicas y también de uñas con esmalte dañado en pabellones quirúrgicos. Se requieren estudios de mejor calidad para abordar estos relevantes tópicos.

Palabras clave: Higiene de manos; infección de herida operatoria; infección del sitio quirúrgico; anillo; uñas; gel de uñas.

\begin{abstract}
Background: Health-care-associated infections are a frequent problem in hospital environments. Hand hygiene is the most effective measure to prevent outbreaks. The use of certain accessories could decrease its effectiveness, facilitating horizontal transmission of pathogens. Objective: Analyze the evidence that assess the impact of the use of rings and nail polish on hand hygiene quality in healthcare workers. Methods: Non-systematic search in PUBMED/ MEDLINE database (1978-2018) of studies in which the quality of hand hygiene or surgical washing is measured, using quantitative cultures or fluorescent stains. Results: Wearing rings: 13 studies met the inclusion criteria. Seven were carried out in general wards. In all of them the use of rings was associated with lower quality of hand hygiene (the majority of low quality). Contrarily, in 3 of 4 primary studies carried out in the operating rooms (of low quality), their use did not affect the quality of surgical washing. Similarly, two systematic reviews obtained similar conclusions. Nail polish: 7 of 54 studies met the inclusion criteria. In four of them there were discordant results (the majority of low quality). One RCT showed a reduction in the quality of surgical washing only when the nail polish was damaged. Gel nail polish was associated with lower quality in two experimental studies. Conclusions: There is insufficient evidence to associate the use of these accessories with the reduction in the quality of hand hygiene. Its safety was not proven neither. Based on the available evidence (the majority of low quality), a negative impact of the use of rings in clinical units and also of damaged nail polish in operating rooms was observed. Better quality studies are required to address these relevant issues.
\end{abstract}

Keywords: Hand hygiene; surgical wound infection; surgical site infections; rings; nails; gel nails. 


\section{Introducción}

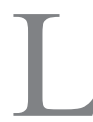

as infecciones asociadas a la atención en salud (IAAS) son una complicación frecuente en el ambiente hospitalario que generan morbilidad, mortalidad, aumento en la estadía hospitalaria y los costos en salud ${ }^{1-5}$. En gran parte son prevenibles mediante medidas efectivas de fácil aplicabilidad y bajo costo ${ }^{6-8}$. Es así, como una adecuada higiene de manos es considerada una medida central para reducir la incidencia de IAAS, ya que las manos son responsables del $80 \%$ de las infecciones hospitalarias ${ }^{9,10}$. Por lo anterior, esta práctica es una de las medidas de prevención más estudiada, efectiva y promovida globalmente ${ }^{11-13}$, a través del desarrollo de innovadoras campañas multimodales, y la permanente vigilancia de potenciales factores que podrían reducir su efectividad ${ }^{14-16}$

Experiencias anteriores revelaron una fuerte asociación entre el uso de uñas artificiales, la portación de microorganismos y la aparición de IAAS ${ }^{17-18}$, lo cual ha abierto la interrogante respecto al uso de uñas esmaltadas y anillos

Tabla 1. Criterios de inclusión considerados para la revisión del impacto del uso de anillos y uñas esmaltadas en el personal de salud

\begin{tabular}{|c|c|}
\hline Diseño del estudio & $\begin{array}{l}\text { Inclusión: } \\
\text { - Revisiones sistemáticas } \\
\text { - ECA controlados y no controlados } \\
\text { - Cohorte prospectivo } \\
\text { - Caso-control retrospectivo } \\
\text { - Experimentales e intervencionales con o sin aleatorización }\end{array}$ \\
\hline Participantes & $\begin{array}{l}\text { Inclusión: personal de la salud, mayor de } 18 \text { años: } \\
\text { - Médicos } \\
\text { - Enfermeras/matronas } \\
\text { - Kinesiólogos } \\
\text { - Estudiantes de medicina, enfermería y kinesiología } \\
\text { - Médicos veterinarios }\end{array}$ \\
\hline \multirow[t]{2}{*}{ Intervención } & $\begin{array}{l}\text { Inclusión: } \\
\text { - Uso de anillos (cualquier tipo) } \\
\text { - Uso de esmalte (convencional y gel) }\end{array}$ \\
\hline & $\begin{array}{l}\text { Exclusión: } \\
\text { - Uso de uñas artificiales }\end{array}$ \\
\hline $\begin{array}{l}\text { Procedimiento de higiene } \\
\text { y lavado quirúrgico de } \\
\text { manos }\end{array}$ & $\begin{array}{l}\text { Inclusión: } \\
\text { - Higiene de manos con: } \\
\text { - Solución alcohólica > 70\% } \\
\text { - Agua y jabón } \\
\text { - Lavado quirúrgico con antiséptico: } \\
\text { - Povidona yodada } \\
\text { - Clorhexidina en base alcohólica }\end{array}$ \\
\hline $\begin{array}{l}\text { Evaluación de la calidad } \\
\text { de la higiene de manos }\end{array}$ & $\begin{array}{l}\text { Métodos aceptados: } \\
\text { - Cultivos cuantitativos } \\
\text { - Tinciones fluorescentes }\end{array}$ \\
\hline
\end{tabular}

ECA: ensayos clínicos aleatorizados. por parte del personal de salud. Se ha planteado que su uso podría reducir la efectividad de la higiene de manos y favorecer la transmisión horizontal de microorganismos ${ }^{19-21}$. Debido a la escasez de ensayos clínicos aleatorizados (ECA), se han generado controversias en los equipos de IAAS y organismos internacionales respecto a permitir el uso de anillos y uñas esmaltadas ${ }^{22}$.

Este artículo tiene como objetivo revisar los estudios que evalúan el impacto del uso de anillos y uñas esmaltadas en la calidad de la higiene de manos de trabajadores de la salud en unidades clínicas y pabellones quirúrgicos.

\section{Metodología}

Se realizó una búsqueda no sistemática en las bases de datos PUBMED /MEDLINE, utilizando la combinación de los términos claves: ("hand hygiene"[Mesh] OR "handwashing" OR "surgical wound infection"[Mesh] OR "surgical site infections") AND "rings". Para la búsqueda de estudios referentes al uso de uñas esmaltadas se utilizó la combinación de los siguientes términos: ("hand hygiene"[Mesh]) OR "hand hygiene" AND "nails" [Mesh] OR "gel nails".

Fueron incluidos estudios observacionales (descriptivos, analíticos), experimentales y revisiones sistemáticas, publicados hasta el 2018. Se limitó la búsqueda a estudios publicados desde 1978, año en el cual se publicaron directrices respecto al lavado quirúrgico de manos por la Asociación de Enfermeras del Quirófano (AORN, por sus siglas en inglés) ${ }^{23}$. Se definió personal de salud a médicos, veterinarios, enfermeras, kinesiológos, técnicos paramédicos y estudiantes del área de la salud. Los estudios que sólo evaluaban el uso de uñas artificiales fueron excluídos, dado que existe consenso de prohibir su uso en el personal de salud ${ }^{11,17,18}$. En base a las recomendaciones de agrupaciones actuales, se aceptaron los estudios en que la higiene de manos fue realizada con solución alcohólica o con agua y jabón, mientras que en el caso del lavado quirúrgico de manos se exigió el uso de algún antiséptico de efectividad demostrada (povidona yodada o clorhexidina en base alcohólica) $)^{11,24,25}$.

Para evaluar la calidad de la higiene de manos se aceptaron los estudios que utilizaron cultivos cuantitativos, los cuales estiman las unidades formadoras de colonias por $\mathrm{ml}$ (ufc/ml). También se incluyeron aquellos realizados con tinciones fluorescentes, dado que han mostrado excelente correlación con los cultivos cuantitativos $(>95 \%)^{26}$ y han sido promovidas en la práctica diaria por su carácter interactivo $^{25}$. Además, de estar disponible, se consignó la tasa de portación de agentes de importancia epidemiológica, tales como Staphylococcus aureus, bacilos gramnegativos (BGN) y Enterococcus spp. Los criterios de inclusión se resumen en la Tabla 1. 


\section{Resultados}

Respecto al impacto del uso de anillos, se encontraron en total 51 artículos. De ellos, 13 no se relacionaban con el tópico, 15 no respondían la pregunta central, tres eran cartas al editor, dos eran revisiones o guías y uno estaba sólo en idioma francés. Tres estudios estaban duplicados o contaban con una versión más actualizada realizada por los mismos autores ${ }^{27-29}$. Hubo dos artículos que no estaban disponibles. En suma, 13 artículos cumplieron con los criterios de inclusión. En cuanto al uso de esmalte de uñas se encontraron en total 54 artículos. De ellos, 26 no se relacionaban con el tópico, 10 no respondían la pregunta central, uno era carta al editor, cinco eran revisiones o guías, tres estaban duplicados o contaban con una versión más actualizada realizada por los mismos autores ${ }^{27-29}$. Finalmente, siete artículos cumplieron con los criterios de inclusión. Considerando las diferencias entre el procedimiento de higiene de manos en unidades clínicas en comparación al lavado quirúrgico de las manos, los resultados de los estudios para el uso de anillos serán presentados en secciones diferentes (Tablas 2 y 3 ). Consecutivamente, los resultados referentes al esmalte tradicional y gel también fueron divididos en secciones diferentes para facilitar el análisis (Tablas 4 y 5).

Tabla 2. Resumen de estudios que evalúan el impacto del uso de anillos sobre la calidad de la higiene de manos en unidades clínicas

\begin{tabular}{|c|c|c|c|c|c|}
\hline $\begin{array}{l}\text { Referencia } \\
\text { (año) }\end{array}$ & $\begin{array}{l}\text { Diseño del } \\
\text { estudio }\end{array}$ & $\begin{array}{l}\text { Tamaño de la } \\
\text { muestra }\end{array}$ & $\begin{array}{l}\text { Herramienta de } \\
\text { medición }\end{array}$ & Unidad & Conclusión \\
\hline $\begin{array}{l}\text { Salisbury, et al. } \\
(1997)^{30}\end{array}$ & $\begin{array}{l}\text { Intervencional, } \\
\text { no aleatorizado }\end{array}$ & 100 participantes & Cultivo cuantitativo & $\begin{array}{l}\text { Unidades clínicas } \\
\text { básicas }\end{array}$ & $\begin{array}{l}\text { Usar anillo se asoció a mayor portación de microorga- } \\
\text { nismos pre y post higiene de manos (BGN y S. aureus) }\end{array}$ \\
\hline $\begin{array}{l}\text { Trick, et al. } \\
(2003)^{31}\end{array}$ & ECA & $\begin{array}{l}564 \text { manos culti- } \\
\text { vadas }\end{array}$ & Cultivo cuantitativo & UPC quirúrgica & $\begin{array}{l}\text { Usar anillo se asoció a mayor portación de microorga- } \\
\text { nismos pre y post higiene de manos (S. aureus, BGN y } \\
\text { Candida sp.) }\end{array}$ \\
\hline $\begin{array}{l}\text { Rupp, et al. } \\
(2008)^{32}\end{array}$ & Observacional & 3.678 mediciones & Cultivo cuantitativo & UPC quirúrgica & $\begin{array}{l}\text { Usar anillo se asoció a mayor portación de microorganis- } \\
\text { mos (incluyendo BGN) }\end{array}$ \\
\hline $\begin{array}{l}\text { Yildrim, et al. } \\
(2008)^{33}\end{array}$ & $\begin{array}{l}\text { Intervencional, } \\
\text { aleatorizado }\end{array}$ & 84 participantes & Cultivo cuantitativo & UPC pediátrica & $\begin{array}{l}\text { Usar anillo se asoció a mayor carga bacteriana post higie- } \\
\text { ne de manos (BGN y bacterias grampositivas) }\end{array}$ \\
\hline $\begin{array}{l}\text { Hautemeniere, et al. } \\
(2010)^{34}\end{array}$ & Observacional & $\begin{array}{l}3.067 \\
\text { participantes }\end{array}$ & Tinción fluorescente & $\begin{array}{l}\text { Unidades básicas } \\
\text { y críticas }\end{array}$ & Usar anillo se asoció a higiene de manos deficiente \\
\hline $\begin{array}{l}\text { Ramón-Cantón, et al. } \\
(2011)^{35}\end{array}$ & Observacional & 293 participantes & Tinción fluorescente & $\begin{array}{l}\text { Unidades básicas } \\
\text { y críticas }\end{array}$ & Usar anillo se asoció a higiene de manos deficiente \\
\hline $\begin{array}{l}\text { Chugh, et al. } \\
(2013)^{36}\end{array}$ & Observacional & 30 participantes & Cultivo cuantitativo & $\begin{array}{l}\text { Unidades clínicas } \\
\text { básicas }\end{array}$ & $\begin{array}{l}\text { Usar anillo se asoció a mayor carga bacteriana post } \\
\text { higiene de manos }\end{array}$ \\
\hline
\end{tabular}

BGN: bacilos gramnegativos, ECA: ensayo clínico aleatorizado, UPC: Unidad de pacientes críticos

\begin{tabular}{|c|c|c|c|c|}
\hline $\begin{array}{l}\text { Referencia } \\
\text { (año) }\end{array}$ & $\begin{array}{l}\text { Diseño del } \\
\text { estudio }\end{array}$ & $\begin{array}{l}\text { Tamaño de la } \\
\text { muestra }\end{array}$ & $\begin{array}{l}\text { Herramienta de } \\
\text { medición }\end{array}$ & Conclusión \\
\hline $\begin{array}{l}\text { Kelsall, et al. } \\
(2006)^{37}\end{array}$ & Observacional & 28 participantes & Cultivo cualitativo & $\begin{array}{l}\text { Usar anillo se asoció a mayor portación de microorganismos pre y post lavado } \\
\text { quirúrgico de manos }\end{array}$ \\
\hline $\begin{array}{l}\text { Waterman, et al. } \\
(2006)^{38}\end{array}$ & $\begin{array}{l}\text { Experimental, } \\
\text { no aleatorizado }\end{array}$ & 19 estudiantes & Cultivo cuantitativo & $\begin{array}{l}\text { Usar anillo no se asoció a mayor portación de microorganismos pre y post } \\
\text { lavado quirúrgico de manos }\end{array}$ \\
\hline $\begin{array}{l}\text { Wongworawat, et al. } \\
(2007)^{39}\end{array}$ & ECA & 20 participantes & Cultivo cuantitativo & $\begin{array}{l}\text { Usar anillo no se asoció a mayor portación de microorganismos pre y post } \\
\text { lavado quirúrgico de manos }\end{array}$ \\
\hline $\begin{array}{l}\text { Al-Allak, et al. } \\
(2008)^{40}\end{array}$ & Observacional & 19 participantes & Cultivo cuantitativo & $\begin{array}{l}\text { Usar anillo no se asoció a mayor portación de microorganismos pre y post } \\
\text { lavado quirúrgico de manos }\end{array}$ \\
\hline $\begin{array}{l}\text { Arrowsmith, et al. } \\
(2014)^{29}\end{array}$ & $\begin{array}{l}\text { Revisión } \\
\text { sistemática }\end{array}$ & $\begin{array}{l}0 \text { participantes } \\
\text { (no encontró } \\
\text { ningún } E C A \text { ) }\end{array}$ & - & $\begin{array}{l}\text { No hay evidencia para asociar el uso de anillos y riesgo de infección del sitio } \\
\text { quirúrgico ni aumento en la carga bacteriana posterior al lavado quirúrgico } \\
\text { de manos }\end{array}$ \\
\hline $\begin{array}{l}\text { Francis, et al. } \\
(2016)^{41}\end{array}$ & $\begin{array}{l}\text { Revisión } \\
\text { sistemática }\end{array}$ & 7 estudios & - & $\begin{array}{l}\text { No hay evidencia suficiente para asociar el uso de anillos con aparición de } \\
\text { infección del sitio quirúrgico en pabellones quirúrgicos }\end{array}$ \\
\hline
\end{tabular}

ECA: ensayo clínico aleatorizado. 


\begin{tabular}{|c|c|c|c|c|c|}
\hline Referencia (año) & $\begin{array}{l}\text { Diseño del } \\
\text { estudio }\end{array}$ & $\begin{array}{l}\text { Tamaño de la } \\
\text { muestra }\end{array}$ & $\begin{array}{l}\text { Herramienta de } \\
\text { medición }\end{array}$ & Unidad & Conclusión \\
\hline $\begin{array}{l}\text { Baumgardner, et al. } \\
(1993)^{42}\end{array}$ & $\begin{array}{l}\text { Experimental, } \\
\text { no aleatorizado }\end{array}$ & 26 participantes & $\begin{array}{l}\text { Cultivo } \\
\text { cuantitativo }\end{array}$ & $\begin{array}{l}\text { Participantes no vinculadas } \\
\text { a la atención clínica }\end{array}$ & $\begin{array}{l}\text { Uñas esmaltadas no se asociaron a mayor carga bacte- } \\
\text { riana post higiene de manos }\end{array}$ \\
\hline $\begin{array}{l}\text { Wynd, et al. } \\
(1994)^{43}\end{array}$ & ECA & 102 enfermeras & $\begin{array}{l}\text { Cultivo } \\
\text { cuantitativo }\end{array}$ & Pabellones quirúrgicos & $\begin{array}{l}\text { Sólo las uñas con el esmalte dañado se asociaron a ma- } \\
\text { yor carga bacteriana post lavado quirúrgico de manos }\end{array}$ \\
\hline $\begin{array}{l}\text { Edel, et al. } \\
(1998)^{44}\end{array}$ & Observacional & 89 participantes & $\begin{array}{l}\text { Cultivo } \\
\text { cuantitativo }\end{array}$ & Pabellones quirúrgicos & $\begin{array}{l}\text { El uso de uñas esmaltadas se asoció a mayor carga de } \\
\text { bacilos gramnegativos posterior al lavado quirúrgico } \\
\text { de manos }\end{array}$ \\
\hline $\begin{array}{l}\text { Arrowsmith, et al. } \\
(2014)^{29}\end{array}$ & $\begin{array}{l}\text { Revisión } \\
\text { sistemática }\end{array}$ & $\begin{array}{c}1 \text { ECA } \\
\text { (102 enfermeras) }\end{array}$ & $\begin{array}{c}\text { Cultivo } \\
\text { cuantitativo }\end{array}$ & Pabellones quirúrgicos & $\begin{array}{l}\text { No hay evidencia suficiente para asociar el uso de uñas } \\
\text { esmaltadas con el deterioro en la calidad del lavado } \\
\text { quirúrgico de manos }\end{array}$ \\
\hline $\begin{array}{l}\text { Hardy, et al. } \\
(2017)^{45}\end{array}$ & $\begin{array}{l}\text { Experimental, } \\
\text { aleatorizado }\end{array}$ & 21 participantes & $\begin{array}{l}\text { Cultivo } \\
\text { cuantitativo }\end{array}$ & $\begin{array}{l}\text { Pabellones quirúrgicos } \\
\text { (cirugía veterinaria) }\end{array}$ & $\begin{array}{l}\text { El uso de uñas esmaltadas no se asoció a mayor carga } \\
\text { bacteriana pre y post lavado quirúrgico de manos }\end{array}$ \\
\hline
\end{tabular}

Tabla 5. Resumen de estudios que evalúan el impacto del uso de uñas con esmalte gel (permanente) sobre la calidad de la higiene de manos

\begin{tabular}{|c|c|c|c|c|c|}
\hline Referencia (año) & $\begin{array}{l}\text { Diseño del } \\
\text { estudio }\end{array}$ & $\begin{array}{l}\text { Tamaño de la } \\
\text { muestra }\end{array}$ & $\begin{array}{l}\text { Herramienta de } \\
\text { medición }\end{array}$ & Unidad & Conclusión \\
\hline $\begin{array}{l}\text { Walaszeq M, et al. } \\
(2018)^{37}\end{array}$ & $\begin{array}{l}\text { Experimental, } \\
\text { aleatorizado }\end{array}$ & 86 participantes & $\begin{array}{c}\text { Cultivo } \\
\text { cuantitativo }\end{array}$ & Sin especificación & $\begin{array}{l}\text { Uso de esmalte gel se asoció a mayor presencia de microor- } \\
\text { ganismos patogénos posterior a la higiene de manos }\end{array}$ \\
\hline $\begin{array}{l}\text { Hewlett A, et al. } \\
(2018)^{38}\end{array}$ & $\begin{array}{l}\text { Esperimental, } \\
\text { no aleatorizado }\end{array}$ & 84 participantes & $\begin{array}{l}\text { Cultivo } \\
\text { cuantitativo }\end{array}$ & Sin especificación & $\begin{array}{l}\text { Uñas con esmalte gel mostraron una menor reducción en la } \\
\text { carga bacteriana posterior a la higiene de manos }\end{array}$ \\
\hline
\end{tabular}

\section{Uso de anillos en Unidades Médico-Quirúrgicas (UMQ) y de Pacientes Críticos (UPC) (Tabla 2)}

Salisbury y cols. observaron en 100 participantes voluntarias de UMQ que el uso de anillos se asociaba a una mayor portación de microorganismos antes y después del lavado de manos medido mediante cultivos cuantitativos. Además, su uso fue asociado a la persistencia de agentes patogénicos (BGN y $S$. aureus) posterior a la higiene de manos. Fue un estudio intervencional, unicéntrico, no aleatorizado, de tamaño muestral reducido y no estimado previamente (50 participantes por grupo). Se controlaron variables tales como el número de anillos, largo de las uñas y la presencia de esmalte ${ }^{30}$. Años más tarde, Trick y cols. diseñaron un ECA para comparar diferentes estrategias de higiene de manos en el personal de salud de una UPC quirúrgica (27 camas). Como desenlace secundario, investigaron el impacto de utilizar anillos mediante un análisis multivariado de 564 manos cultivadas antes y después de realizar higiene de las mismas. Observaron que los funcionarios que utilizaban anillo presentaban 10 veces más riesgo de portar algún microorganismo (incluyendo $S$. aureus, BGN y Candida spp.). Este riesgo persistió independientemente de la estrategia de higiene de manos (solución jabonosa, toalla con químico antimicrobiano o solución alcohólica) o al tipo de anillo utilizado (liso o con piedra) (OR: 2,6), y aumentaba si se portaba más de un anillo (OR: 4,6) ${ }^{31}$. Se trató de un ECA, unicéntrico, controlado, de adecuado tamaño (564 mediciones), pero que abordó el uso de anillos en forma indirecta mediante un subanálisis. Posteriormente, Rupp y cols. diseñaron un estudio para comparar dos estrategias de higiene de manos en dos UPC quirúrgicas de tamaño y características similares (12 camas cada una). Observaron mayor portación de BGN $(p=0,049)$ y microorganismos en general $(p=0,002)$ en trabajadores de salud que portaban anillos ${ }^{32}$. Fue un estudio observacional, prospectivo y controlado, de adecuado tamaño muestral (3678 oportunidades de medición). Su principal limitación es que los cultivos cuantitativos se realizaban cada 60 días y no necesariamente posterior a la higiene de manos. Por lo anterior, sólo sugiere mayor portación de agentes bacterianos durante la jornada de trabajo, pudiendo no coincidir con el momento posterior a la higiene de manos. Yildrim y cols. evaluaron en una UPC pediátrica a enfermeras designadas aleatoriamente a utilizar anillo liso $(\mathrm{n}=28)$, anillo con piedra $(n=28)$, en comparación a aquellas que 
no lo usaban $(\mathrm{n}=28)$. Posterior a la higiene de manos (solución alcohólica), observaron mayor cantidad de bacterias grampositivas y BGN en aquellos participantes que portaban anillo $(\mathrm{p}=0,001)$. No hubo diferencias entre el tipo de anillo utilizado (liso o con piedra) ${ }^{33}$. En este estudio intervencional, de tamaño muestral reducido, los grupos fueron designados aleatoriamente. Fueron controladas algunas variables de confusión, tales como el uso de esmalte de uñas y de antimicrobianos recientes. No fue controlado el largo de las uñas. Hautemeniere A, y cols. evaluaron la efectividad de la higiene de manos (solución alcohólica) en relación a la implementación de un programa de educación y reforzamiento de la técnica. Tras la intervención, se observó una mejoría sostenida en la calidad en la higiene de manos medida con tinción fluorescente. No obstante, el uso de anillo se asoció a una reducción en la calidad de la higiene de manos durante el seguimiento (OR: 2,0) ${ }^{34}$. Corresponde a un estudio unicéntrico, de gran tamaño (3067 participantes), con una fase inicial observacional-prospectiva, y una segunda fase intervencional, no aleatoria, pero se controlaron algunas variables relevantes (duración de la higiene de manos y largo de las uñas). En esa misma línea, en otro estudio realizado en UMQ y UPC se observó, mediante la aplicación de un agente fluorescente, que $84 \%$ del personal que utilizaba anillo presentaba deficiencias en la higiene de los dedos de las manos, respecto a $46 \%$ que no lo usaba $(\mathrm{p}<0,001)^{35}$. Se trató de un estudio observacional, prospectivo no controlado, de adecuado tamaño muestral (295 participantes). No fue estandarizada la técnica ni duración del procedimiento de higiene de manos. Finalmente, un estudio realizado en estudiantes de medicina observó, mediante mediciones de cultivos cuantitativos, mayor cantidad de microorganismos tras la higiene de manos en aquellos estudiantes que usaban anillo $(p=0,05)$. Se trató de un pequeño estudio observacional $(n=30)$, sin control sobre variables de confusión ${ }^{36}$.

\section{Uso de anillos en pabellones quirúrgicos (Tabla 3)}

La necesidad de remoción de los anillos previo al lavado quirúrgico de manos ha sido también evaluada. Kesal y cols. observaron en cirujanos y enfermeras de pabellón mayor portación de microorganismos en usuarios de anillos, tanto previa $(\mathrm{p}=0,05)$ como posteriormente al lavado quirúrgico (gluconato de clorhexidina 0,5\%) $(p=0,025)$. Los participantes que se removían el anillo momentos antes del lavado, mantenían una mayor carga bacteriana con respecto a los que nunca lo portaban $(\mathrm{p}=0,05)^{37}$. Se trató de un estudio observacional, de pequeño tamaño (28 participantes). A diferencia de otros estudios, utilizaron la mano contralateral como grupo control, no pudiendo descartar el traspaso horizontal de microorganismos durante el lavado de manos. En contraste, Waterman y cols. realizaron un estudio en estudiantes de medicina veterinaria, los cuales eran designados a usar $(n=11)$ o no usar anillo $(n=8)$. Todos portaban uñas menores a $5 \mathrm{~mm}$ de largo. Se cuantificó la carga bacteriana al inicio del estudio y tres horas posterior al lavado de manos quirúrgico (gluconato de clorhexidina), período en el cual realizaron disecciones de cadáveres de perros. El análisis comparativo de los cultivos cuantitativos no demostró significancia estadística cuando se analizaron todas las manos con anillo versus todas las manos sin anillo pre y post lavado quirúrgico de $\operatorname{manos}^{38}$. Este estudio intervencional, comparativo, de reducido tamaño muestral $(n=19)$, no tuvo aleatorización ni control de variables de confusión. Wongworawat y cols. diseñaron un ECA pequeño (20 participantes por grupo), en el que observaron una mayor efectividad del lavado quirúrgico de manos en el grupo que utilizó clorhexidina alcohólica $(n=20$ participantes). De un subanálisis, no encontraron diferencias entre aquellos que portaban o no anillo ${ }^{39}$. Corresponde a un ECA controlado, no enmascarado, de limitado tamaño muestral y diseñado para comparar diferentes métodos de lavado quirúrgico de manos, y que en forma secundaria se realizó un subanálisis del impacto de la portación de anillos en cada grupo (manos con y sin anillos). No se describen las características basales de los participantes ni tampoco se menciona si existieron diferencias entre los diferentes grupos comparados, tales como la edad, género o el largo de las uñas. Otro estudio, realizado por A-Allak y cols., evaluó a anestesistas y cirujanos que llevaban un anillo liso (mano izquierda) durante el lavado quirúrgico de manos. Posteriormente, éste se retiraba y mediante una tórula estéril se cultivaba su circunferencia interna y las manos de los participantes en placas de agar sangre. Todas las muestras de las manos resultaron positivas para algún agente infeccioso, pero no se observaron diferencias significativas entre las manos derechas e izquierdas de los cirujanos $(p=0,26)$ ni de los anestesistas $(\mathrm{p}=0,383)^{40}$. Corresponde a un estudio observacional, de pequeño tamaño muestral (19 participantes). El grupo "control" fueron las manos opuestas de los mismos participantes pudiendo haber transmisión horizontal de microorganismos de una mano a otra durante el lavado quirúrgico. Existen dos revisiones sistemáticas que abordan también este tópico. La primera, fue una realizada por el Grupo Colaborativo Cochrane, la cual tuvo como objetivo evaluar el impacto del uso de anillos y uñas esmaltadas en la calidad del lavado quirúrgico de manos en ECA. Ningún estudio cumplió con los criterios de inclusión, por lo cual concluye que no existe evidencia para asociar un impacto negativo del uso de anillos en el lavado quirúrgico de $\operatorname{manos}^{29}$. La segunda revisión sistemática fue realizada por Francis y cols., quienes incluyeron siete estudios que evaluaban el uso de anillos en pabellones quirúrgicos, concluyendo que no existe evidencia suficiente para asociar el uso de este 
accesorio con el aumento de infecciones del sitio quirúrgico ni aumento del recuento bacteriano ${ }^{41}$. Esta revisión sistemática fue amplia, e incluyó las características de los estudios incluidos. Sin embargo, no realizó un análisis de la calidad de éstos (en su mayoría estudios de baja calidad) incluyendo algunos que no evaluaban directamente el impacto respecto al lavado quirúrgico de manos. Tampoco se conocen los estudios que fueron excluídos y si se incluyó en la búsqueda literatura científica "gris".

\section{Uso de esmalte de uñas en el ambiente hospitalario en general (Tabla 3)}

Una de las primeras aproximaciones fue reportada por Baumgardner y cols. Estudiaron a 26 mujeres sanas con uñas limpias y cortas. Se tomaron cultivos de las uñas al comienzo del estudio y luego fueron designadas a utilizar esmalte de uñas en una de sus manos, sirviendo la otra mano como control. Mantuvieron el esmalte durante cuatro días, en los cuales se realizaron cultivos diarios pre y post-higiene de manos con agua y jabón, sin observar diferencias significativas en el recuento bacteriano (ufc/ $\mathrm{ml})$ entre las manos con y sin esmalte $(\mathrm{p}>0,01)^{42}$. Se trató de un estudio intervencional no aleatorizado, de pequeño tamaño muestral. El diseño del estudio impidió comparar entre aquellos con esmalte indemne y dañado, dado que al cuarto día, todos los participantes salvo uno, presentaban daño en el esmalte. Wynd y cols. realizaron un ECA, en el cual designaron aleatoriamente a 102 enfermeras de pabellón a utilizar uñas con esmalte indemne $(<2$ días, $n=34$ ), uñas con esmalte dañado (daño visible o $>4$ días, $\mathrm{n}=34$ ) y uñas naturales ( $\sin$ esmalte, $\mathrm{n}=34$ ). La técnica del lavado fue estrictamente supervisada con una alta tasa de cumplimiento (92\%). Los cultivos obtenidos antes y después del lavado quirúrgico (realizado con povidona yodada) no revelaron una mayor carga bacteriana para el grupo con esmalte indemne con respecto a aquellos que no utilizaban esmalte $(\mathrm{p}>0,05)$. El esmalte dañado se asoció significativamente a mayor carga bacteriana posterior a la higiene de manos $(\mathrm{p}<0,035)$. Se trató de un ECA, aleatorizado, de adecuado tamaño muestral, en el que se controló el largo de las uñas y la técnica del lavado quirúrgico de manos ${ }^{43}$. En otro estudio, Edel y cols. realizaron cultivos pre y post-lavado quirúrgico en 89 trabajadoras experimentadas de pabellón quirúrgico (enfermeras, cirujanos, anestesistas, entre otros). Fueron capacitadas y supervisadas para realizar una adecuada técnica de lavado quirúrgico de manos. El uso de esmalte de uñas se asoció a mayor colonización por BGN posterior al lavado quirúrgico de manos $(\mathrm{p}=0,0008)^{44}$. Se trató de un estudio observacional, de adecuado tamaño, en que fue controlado el largo de las uñas y la técnica del lavado quirúrgico de manos. Más recientemente, Hardy y cols. midieron el recuento bacteriano pre y post-lavado quirúrgico de manos (clorhexidina 2\%) en 21 trabajadores de cirugía veterinaria que fueron designados en forma aleatoria a usar uñas esmaltadas. Se tomaron cultivos posterior al término de la cirugía y se controló el largo de las uñas. No se observaron diferencias significativas en la carga bacteriana en los participantes con uñas esmaltadas con respecto a los controles $(\mathrm{p}>0,05)$. Tampoco su uso fue asociado a una mayor carga bacteriana en el análisis multivariado $(\mathrm{p}=0,45)^{45}$. Se trató de un estudio experimental, de reducido tamaño muestral $(n=21)$ y ausencia de control sobre la técnica de lavado quirúrgico de manos (sólo se controló su duración).

Finalmente, la revisión sistemática realizada por el Grupo Colaborativo Cochrane comentada anteriormente, evaluó el impacto del uso de anillos y uñas esmaltadas en la calidad del lavado quirúrgico de manos. La búsqueda incluyó sólo ensayos clínicos aleatorios, encontrando sólo un artículo respecto al uso de uñas esmaltadas (Wynd 1994), el cual fue analizado previamente. En base a éste, se concluye que no existe evidencia suficiente para asociar el uso de uñas con esmalte en buen estado al deterioro en la calidad del lavado de $\operatorname{manos}^{29}$.

\section{Uso de uñas con esmalte gel (esmalte permanente) (Tabla 4)}

El esmalte gel está constituido en base de monoacrilato, el cual tras secarse bajo luz ultravioleta permite que el barniz se adhiera con mayor firmeza a la uña, permaneciendo por más tiempo ${ }^{46}$. Un estudio piloto evaluó la efectividad de la higiene de manos en relación a distintos tipos de esmaltes y largo de las uñas. Se comparó a personal de salud con uñas naturales $(n=45)$ con cuatro grupos experimentales: esmalte tradicional $(n=10)$, sólo barniz $(\mathrm{n}=11)$, barniz híbrido con luz UV $(\mathrm{n}=15)$ y esmalte gel con luz UV $(\mathrm{n}=7)$. Post higiene de manos se realizaron cultivos cualitativos de uña y área periungueal buscando agentes infecciosos comensales y patógenos. Tanto el largo de las uñas, como la presencia de esmalte gel se asociaron en forma independiente a mayor persistencia de microorganismos potencialmente patógenos tras la higiene de manos (esmalte gel, OR: 9,2) ${ }^{47}$. Se trató de un estudio experimental, aleatorizado, de reducido tamaño en cada grupo, en el cual se controló el largo de las uñas. En otro estudio piloto, Hewlett y cols. compararon uñas naturales (control), con esmalte tradicional y con esmalte gel. A un total de 88 participantes, se les realizaron cultivos cuantitativos (ufc/ml) de uñas y dedos al día 1, 7 y 14 post-inicio del estudio. Al día 7 se les realizó cultivo pre y post-higiene de manos (con alcohol gel). Hubo significativamente menor reducción de la carga bacteriana posterior a la higiene de manos en aquellos que usaban esmalte gel respecto a los usuarios de uñas no esmaltadas ${ }^{48}$. Se trató de un estudio experimental, sin especificar el tamaño de la muestra, sin aleatorización ni control sobre la técnica de higiene de manos. 


\section{Discusión}

Con respecto al uso de anillos en unidades clínicas y el riesgo de IAAS, no existe evidencia de calidad suficiente para responder esta interrogante. Sin embargo, en los siete estudios encontrados se observó en forma consistente reducción en la calidad de la higiene de manos al utilizar este accesorio ${ }^{30-36}$. En algunos, el uso de anillo fue asociado a agentes infecciosos de importancia epidemiológica, tales como $S$. aureus y $\mathrm{BGN}^{31-33}$. Todos los estudios poseen importantes limitaciones metodológicas. Tampoco existe evidencia suficiente para asociar el uso de anillos en pabellones quirúrgicos y un mayor riesgo de IAAS. A diferencia de su uso en unidades clínicas, en los pabellones quirúrgicos no se advierte una reducción en la calidad del lavado quirúrgico de manos en la mayoría de los estudios analizados ${ }^{29,37-41}$. No obstante, todos los estudios primarios poseen importantes limitaciones metodológicas. Referente a las dos revisiones sistemáticas, una fue construida sólo en base a estudios de alta calidad (sin hallazgos en los resultados de la búsqueda $)^{29}$ y la segunda realizada en base a estudios mayoritariamente de baja calidad ${ }^{41}$. Estos resultados podrían explicarse por las características del lavado de manos quirúrgico, el cual a diferencia del lavado clínico de manos es más acucioso, utiliza siempre un antiséptico y posee mayor tiempo de duración ${ }^{24}$. A pesar de lo anterior, se han reportado experiencias negativas, como la de Jepson y cols. que reportaron un brote de $S$. marsascens en seis pacientes con infección de esternotomía, en el cual se aisló el agente en el anillo del cirujano. Tras la remoción del accesorio, no se observaron nuevos $\operatorname{casos}^{49}$. En la actualidad grupos de expertos de asociaciones internacionales siguen recomendando la remoción de joyas previo al lavado de manos quirúrgico ${ }^{11,23,24,51}$.

Con respecto al uso de uñas esmaltadas, el ECA de mejor calidad no demostró mayor carga bacteriana asociado al uso de uñas esmaltadas tras el lavado quirúrgico de manos, salvo cuando éste se encontraba dañado ${ }^{43}$. Lo anterior refuerza que el estado del esmalte podría ser relevante y que su impacto sobre el lavado quirúrgico de manos podría ser menor dadas las características comentadas. En forma adicional, el resto de los estudios (de menor calidad), muestran resultados contradictorios respecto al esmalte tradicional, impidiendo sacar conclusiones definitivas.

Del mismo modo, tampoco hay evidencia suficiente para asociar el uso de esmalte gel a un mayor riesgo de IAAS. Se encontraron sólo dos estudios experimentales y de baja calidad ${ }^{47,48}$. En ambos se observó una reducción en la calidad en la higiene de manos. Esto podría explicarse por microtraumas en la uña especialmente durante el proceso de remoción, el cual se ha asociado a debilidad, adelgazamiento, fragilidad, pseudoleuconiquia y onicosquicia. De forma concomitante, pueden producir reacciones de dermatitis periungueal por contacto, facilitando la portación de microorganismos ${ }^{46}$.

\section{Opinión de sociedades y agrupaciones internacionales respecto al esmalte gel}

La AORN ha manifestado que aún no podemos saber si el uso de estos esmaltes podrían aumentar el riesgo de portar patógenos tal como las uñas artificiales, por lo cual sugiere abordar el tema a nivel multidisciplinario en cada institución ${ }^{24}$. La Asociación de Profesionales en Control de Infecciones y Epidemiología (APIC) se ha manifestado en la misma dirección ${ }^{50}$, mientras que la Organización Mundial de Salud (OMS) no hace referencia específica al esmalte gel, pero enfatiza en mantener las uñas en su forma natural y cortas ${ }^{11}$. La Sociedad Americana de Epidemiología Hospitalaria (SHEA, por sus siglas en inglés) afirma que ante la ausencia de evidencia específica para uñas con esmalte gel, se debe considerar un enfoque conservador (tratándolas como uñas artificiales), evitando su uso en áreas de pacientes de alto riesgo de infección ${ }^{24}$.

\section{Opinión de los autores}

Se comprueba que existe escasa evidencia y en su gran mayoría con importantes limitaciones metodológicas que dificultan concluir definitivamente respecto a los tópicos discutidos. Se comentan algunas consideraciones al respecto:

- No existe evidencia suficiente que respalde la inocuidad del uso de uñas esmaltadas y anillos.

- En la práctica hospitalaria, es muy difícil realizar una vigilancia del estado del esmalte en el personal de salud, debido al insuficiente recurso humano para garantizar una supervisión efectiva. Con muchas dificultades los equipos de IAAS logran supervisar y promover la adherencia a las medidas básicas en prevención ${ }^{51}$.

- Si la higiene de manos, que es una medida efectiva, universalmente aceptada y promovida, posee una adherencia baja y heterogénea entre el personal de salud $^{52-57}$, es válido cuestionarse cómo será la adherencia a la mantención "en buen estado" de las uñas esmaltadas.

- La atención clínica sin anillos ni uñas esmaltadas es una medida segura, sin costo, y sencilla de implementar.

\section{Conclusiones}

No existe evidencia de calidad y de consistencia suficiente para asociar el uso de anillos y esmalte para uñas (tradicional y gel) a una reducción en la calidad de la higiene de manos. Tampoco está probada su inocuidad. 


\section{Whilum Infecciones Asociadas a la Atención de Salud}

Se requiere incentivar a los equipos de IAAS a realizar más estudios y de mejor calidad metodológica para resolver estas interrogantes. Por ahora, cualquier conducta que pueda potencialmente disminuir la efectividad de la higiene de manos debiera evitarse, más aún cuando son prácticas sencillas de instaurar.

\section{Referencias bibliográficas}

1.- Allegranzi B, Bagheri Nejad S, Combescure C, Graafmans W, Attar H, Donaldson L, et al. Burden of endemic health-care-associated infection in developing countries: systematic review and meta-analysis. Lancet 2011; 377: 228-41. doi: 10.1016/S0140-6736(10)61458-4.

2.- Indicadores de referencia de infecciones asociadas a la atención en salud vigenetes desde Mayo de 2017. Circular C37 nº 04. Subsecretaría de redes asistencias, Ministerio de Salud, Chile. Disponible en: http://web. minsal.cl/wp-content/uploads/2017/11/ CIRCULAR-ENVIA-INDICADORES-DEREFERENCIA-DE-IAAS-para-2017-corr.pdf.

3.- Anderson D J, Kaye K S, Chen L F, Schmader K E, Choi Y, Sloane R, et al. Clinical and financial outcomes due to methicillin resistant Staphylococcus aureus surgical site infection: a multi-center matched outcomes study. PLoS One 2009; 4: e8305. doi: 10.1371/journal. pone. 0008305 .

4.- Vergara T, Fica A. Estudio de costo de las infecciones del torrente sanguíneo asociadas a catéter vascular central en pacientes adultos en Chile. Rev Chilena Infectol 2015; 32: 634-8. doi: $10.4067 / \mathrm{S} 0716-10182015000700004$.

5.- Véliz E, Fica A. Costo de las neumonías asociadas a la ventilación mecánica en pacientes adultos en un hospital general en Chile. Rev Chilena Infectol 2017; 34: 447-52. doi: 10.4067/S0716-10182017000500447.

6.- Pittet D, Boyce J. Hand hygiene and patient care: pursuing the Semmelweis legacy. Lancet Infect Dis 2001; 1: 9-20. doi: 10.1016/S14733099(09)70295-6.

7.- Pittet D. Improving adherence to hand hygiene practice: a multidisciplinary approach. Emerg Infect Dis. 2001; 7: 234-40.

8.- Pittet D, Allegranzi B, Sax H, Dharan S, Pessoa-Silva L, Donaldson L, et al. Evidencebased model for hand transmission during patient care and the role of improved practices. Lancet Infect Dis 2006; 6: 641-52.

9.- Kampf G, Kramer A. Epidemiologic background of hand hygiene and evaluation of the most important agents for scrubs and rubs. Clin Microbiol Rev 2004; 17: 863-93.

10.- Kampf G, Löffler H, Gastmeier P. Hand hygiene for the prevention of nosocomial infections. Dtsch Arztebl Int 2009; 106: 649-55. doi: 10.3238/arztebl.2009.0649.

11.- World Health Organisation (WHO). WHO
Guidelines on Hand Hygiene in Health Care. Geneva, Switzerland; World Health Organization, 2009. 270 p. Disponible en: https://apps.who.int/iris/bitstream/ handle/10665/44102/9789241597906_eng. pdf;sequence $=1$.

12.- Noakes TD, Borrensen J, HewButler T, Lambert MI, Jordaan E. Semmelweis and the aetiology of puerperal sepsis 160 years on: an historical review. Epidemiol Infect 2008; 136: $1-9$.

13.- Saito H, Allegranzi B, Pittet D. 2018 WHO hand hygiene campaign: preventing sepsis in health care and the path to universal health coverage. Lancet Infect Dis 2018;18: 490-2. doi: 10.1016/S1473-3099(18)30228-7.

14.- Ling M L, How K B. Impact of a hospital-wide hand hygiene promotion strategy on healthcareassociated infections. Antimicrob Resist Infect Control 2012; 1: 13. doi: 10.1186/2047-2994-113.

15.- Aboumatar H, Ristaino P, Davis R O, Thompson C B, Maragakis L, Cosgrove S, et al. Infection prevention promotion program based on the PRECEDE model: improving hand hygiene behaviors among healthcare personnel. Infect Control Hosp Epidemiol 2012; 33: 14451. doi: $10.1086 / 663707$.

16.- Nobile M, Conti C, Bastianelli A, Piscitelli A, Calori G M, Navone P. Promotion of hand hygiene: the experience of the orthopaedic hospital Gaetano Pini-CTO, Milan, Italy. Ann Ig 2018; 30: 229-36. doi:10.7416/ai.2018.2214.

17.- Porteous J. Artificial nails... very real risks. Can Oper Room Nurs J 2002; 20: 16-7, 20-1.

18.- Toles A. Artificial nails: are they putting patients at risk? A review of the research. J Pediatr Oncol Nurs 2002; 19: 164-71.

19.- Fagernes M, Lingaas E, Bjark P. Impact of a single plain finger ring on the bacterial load on the hands of healthcare workers. Infect Control Hosp Epidemiol 2007; 28: 1191-5.

20.- Fagernes M, Lingaas E. Impact of finger rings on transmission of bacteria during hand contact. Infect Control Hosp Epidemiol 2009; 30: 42732. doi: $10.1086 / 596771$.

21.- Fagernes M, Lingaas E. Factors interfering with the microflora on hands: a regression analysis of samples from 465 healthcare workers. J Adv Nurs 2011; 67: 297-307. doi: 10.1111/j.13652648.2010.05462.x.

22.- Cimon K, Featherstone R. Jewellery and nail polish worn by health care workers and the risk of infection transmission: a review of clinical evidence and guidelines. Ottawa $(\mathrm{ON})$ : Canadian Agency for Drugs and Technology in Health (CADTH); 2017 Mar.

23.- Standards for surgical hand scrubs, in AORN Standards of Practice (Denver: Association of Operating Room Nurses, Inc, 1978: 3-23.

24.- Golberg J L. Guideline Implementation: Hand Hygiene. AORN J 2017; 105: 203-212. doi: 10.1016/j.aorn.2016.12.010.

25.- Ellingson K, Haas J, Aiello A, Kusek L, Maragakis L, Olmsted R, et al. Strategies to prevent healthcare-associated infections through hand hygiene. Infect Control Hosp Epidemiol 2014; 35: 937-60. doi: 10.1086/677145.

26.- Lehotsky Á, Szilágyi L, Bánsághi S, Szerémy P, Wéber G, Haidegger T. Towards objective hand hygiene technique assessment - Validation of the UV dye based hand rubbing quality assessment procedure. J Hosp Infect 2017; 97 : 26-9. doi: 10.1016/j.jhin.2017.05.022.

27.- Arrowsmith V A, Maunder J A, Sargent R J, Taylor R. Removal of nail polish and finger rings to prevent surgical infection. Cochrane Database Syst Rev. 2001: CD003325.

28.- Arrowsmith V A, Taylor R. Removal of nail polish and finger rings to prevent surgical infection. Cochrane Database Syst Rev 2012: CD003325.

29.- Arrowsmith V A, Taylor R. Removal of nail polish and finger rings to prevent surgical infection. Cochrane Database Syst Rev 2014: CD003325.

30.- Salisbury D M, Hutfilz P, Treen L M, Bollin G $\mathrm{E}$, Gautam S. The effect of rings on microbial load of health care workers' hands. Am J Infect Control 1997; 25: 24-7.

31.- Trick W E, Vernon M O, Hayes R A, Nathan C, Rice W T, Peterson B J, et al. Impact of ring wearing on hand contamination and comparison of hand hygiene agents in a hospital. Clin Infect Dis 2003; 36: 1383-90.

32.- Rupp M E, Fitzgerald T, Puumala S, Anderson J R, Craig R, Iwen P C, et al. Prospective, controlled, cross-over trial of alcoholbased hand gel in critical care units. Infect Control Hosp Epidemiol 2008; 29 (1): 8-15. doi: 10.1086/524333.

33.- Yildirim I, Ceyhan M, Cengiz A B, Bagdat A, Barin C, Kutluk T, et al. A prospective comparative study of the relationship between different types of ring and microbial hand colonization among pediatric intensive care unit nurses. Int J Nurs Stud 2008; 45: 1572-6. 
doi: 10.1016/j.ijnurstu.2008.02.010.

34.- Hautemaniere A, Cunat L, Diguio N, Vernier N, Schall C, Daval M C. Factors determining poor practice in alcoholic gel hand rub technique in hospital workers. J Infect Public Health 2010; 3: 25-34. doi: 10.1016/j.jiph.2009.09.005.

35.- Ramón-Cantón C, Boada-Sanmartín N, Pagespetit-Casas L. Evaluación de la técnica de higiene de manos en profesionales asistenciales. Rev Calidad Asistencial 2011; 26: 376-9. DOI: 10.1016/j.cali.2011.09.002

36.- Chugh Y, Baligathe S. Semmelweis's forgotten gift: Has handwashing lost its importance?. J Clin Diagn Res 2013; 7: 434-6. doi: 10.7860/ JCDR/2013/4779.2792.

37.- Kelsall N K, Griggs R K, Bowker K E, Bannister G C. Should finger rings be removed prior to scrubbing for theatre? J Hosp Infect 2006; 62: 450-2.

38.- Waterman T R, Smeak D D, Kowalski J, Hade E M. Comparison of bacterial counts in glove juice of surgeons wearing smooth band rings versus those without rings. Am J Infect Control 2006; 34: 421-5.

39.- Wongworawat M D, Jones S G. Influence of rings on the efficacy of hand sanitation and residual bacterial contamination. Infect Control Hosp Epidemiol 2007; 28: 351-3.

40.- Al-Allak A, Sarasin S, Key S, Morris-Stiff G. Wedding rings are not a significant source of bacterial contamination following surgical scrubbing. Ann R Coll Surg Engl 2008; 90: 133-5. doi: 10.1308/003588408X242051.

41.- Francis R H, Mudery J A, Tran P, Howe C, Jacob A. The case for using evidence-based guidelines in setting hospital and public health policy. Front Surg 2016; 3: 20. doi: 10.3389/ fsurg.2016.00020.

42.- Baumgardner C, Maragos C, Walz M, Larson E. Effects of nail polish on microbial growth of fingernails. Dispelling sacred cows. AORN J
1993; 58: 84-8.

43.- Wynd C A, Samstag D E, Lapp A M. Bacterial carriage on the fingernails of OR nurses. AORN J 1994; 60: 799-805.

44.- Edel E, Houston S, Kennedy V, LaRocco M. Impact of a 5-minute scrub on the microbial flora found on artificial, polished, or natural fingernails of operating room personnel. Nursing Research 1998; 47: 54-9.

45.- Hardy J M, Owen T J, Martinez S A, Jones L P, Davis M A. The effect of nail characteristics on surface bacterial counts of surgical personnel before and after scrubbing. Vet Surg 2017; 46: 952-61. doi: 10.1111/vsu. 12685 .

46.- Rieder E A, Tosti A. Cosmetically induced disorders of the nail with update on contemporary nail manicures. J Clin Aesthet Dermatol 2016; 9: 39-44.

47.- Walaszeq M, Kolpa M, Różańska A, Jagiencarz-Starzec B, Wolak Z, WójkowskaMach J. Nail microbial colonization following hand disinfection: a qualitative pilot study. J Hosp Infect 2018; 100: 207-10. doi: 10.1016/j. jhin.2018.06.023.

48.- Hewlett A, Hohenberger H, Murphy C N, Helget L, Hausmann H, Lyden E, et al. Evaluation of the bacterial burden of gel nails, standard nail polish, and natural nails on the hands of health care workers. Am J Infect Control 2018; 46: 1356-9. doi: 10.1016/j. ajic.2018.05.022.

49.- Jepson A P, McDougall C, Clark A, Bateman A, Williamson G, Kaufmann M E. Finger rings should be removed prior to scrubbing (Letters to the Editor). J Hosp Infect 2006; 64: 197-8.

50.- Association for Professionals in Infection Control and Epidemiology (APIC). Practice Resources. Disponible en: http://www.apic. org/Professional-Practice/Practice-Resources. (Fecha de acceso: 24 de octubre 2018)

51.- Jarrin Tejada C, Bearman G. Hand hygiene compliance monitoring: the state of the art. Curr Infect Dis Rep. 2015; 17: 470. doi: 10.1007/s11908-015-0470-0.

52.- Barrett R, Randle J. Hand hygiene practices: nursing students' perceptions. J Clin Nurs 2008; 17: 1851-7. doi: 10.1111/j.13652702.2007.02215.x.

53.- Erasmus V, Brouwer W, van Beeck E F, Oenema A, Daha T J, Richardus J H, et al. A qualitative exploration of reasons for poor hand hygiene among hospital workers: lack of a positive role model and of convincing evidence that hand hygiene prevents cross-infections. Infect Control Hosp Epidemiol 2009; 30: 4159. doi: 10.1086/596773.

54.- Rosenthal V D, Pawar M, Leblebicioglu H, Navoa-Ng J A, Villamil-Gómez W, ArmasRuiz A, et al. Impact of the International Nosocomial Infection Control Consortium (INICC) multidimensional hand hygiene approach over 13 years in 51 cities of 19 limited-resource countries from Latin America, Asia, the Middle East, and Europe. Infect Control Hosp Epidemiol 2013; 34: 415-23. doi: 10.1086/669860.

55.- Azim S, McLaws M L. Doctor, do you have a moment? National Hand Hygiene Initiative compliance in Australian hospitals. Med J Aust 2014; 200: 534-7.

56.- Mumford V, Greenfield D, Hogden A, Debono D, Gospodarevskaya E, Forde K, et al. Disentangling quality and safety indicator data: a longitudinal, comparative study of hand hygiene compliance and accreditation outcomes in 96 Australian hospitals. BMJ Open 2014; 4: e005284. doi: 10.1136/bmjopen-2014-005284.

57.- Smiddy M P, O' Connell R, Creedon S A. Systematic qualitative literature review of health care workers' compliance with hand hygiene guidelines. Am J Infect Control 2015; 43: 269- 74. doi: 10.1016/j.ajic.2014.11.007. 